\title{
Les problèmes méthodologiques de la mesure des impacts économiques locaux des universités
}

\section{The methodological problems of measurement of local economic impact of universities}

\author{
Balázs KoTosz \\ Université de Szeged \\ kotosz@eco.u-szeged.hu \\ Auteur correspondant \\ Marie-France GAUNARD-ANDERSON \\ Université de Lorraine \\ marie-france.gaunard@univ-lorraine.fr \\ Miklós LuKovics \\ Université de Szeged \\ miki@eco.u-szeged.hu
}

Mots-clés : étude d'impact, France, Hongrie, universités

Keywords : France, Hungary, impact study, universities Classification JEL : H44, I23, I25, R12, R53 


\section{Résumé}

L'impact économique local d'une institution d'enseignement supérieure comme une université est une question qui a été largement abordée dans la littérature. L'étude d'impact économique est devenue également un outil standard utilisé à faire prendre conscience les États de l'importance des dépenses dans l'enseignement supérieur. La définition la plus générale est "la différence entre l'activité économique existant dans une région dans le cas de la présence de l'institution et le niveau d'activité qui aurait été présent si l'institution n'existait pas. "Dans la pratique, il y a une série des problèmes importants : la séparation de l'effet net et brut, l'identification des missions de l'université, le choix de l'échelle territoriale, le choix du modèle statistique, l'estimation des impacts induits et catalytiques, etc. Après l'introduction théorique des problèmes méthodologiques, une analyse comparative de l'Université de Szeged (Hongrie) et de l'Université de Lorraine (France) sera présentée. Une analyse des limites est aussi esquissée.

\section{Abstract}

Nowadays the realization that certain economic units, universities or other objects have impact on the economy of their region comes more and more into prominence. The economic impact study has become a standard tool to persuade state legislatures of the importance of expenditures on higher education. The most general definition is as "the difference between existing economic activity in a region given the presence of the institution and the level that would have been present if the institution did not exist." In the practice we face a series of problems: separation of net and gross impact, identification of universities' missions, territorial level choice, statistical model choice, estimation of induced and catalytic impacts, etc. After a methodological review, we demonstrate the example of the University of Szeged (Hungary) and the University of Lorraine (France). An analysis of the limitations is also provided. 


\section{- 1 - \\ Introduction}

L'impact économique local d'une institution d'enseignement supérieur ${ }^{1}$ comme une université est une question qui a attiré une attention considérable dans la littérature. Веск et al. (1995) définissent l'impact économique comme étant «la différence entre l'activité économique existant dans une région dans le cas de la présence de l'institution et le niveau d'activité qui aurait été présent si l'institution n'existait pas. » En général, il y a trois problèmes importants. Le premier est celui de la définition de l'impact (net ou brut ; direct, indirect, induit, catalytique) ; puis il s'agit de mesurer et d'estimer les flux et les séparer selon les missions universitaires ; enfin, il faut procéder à l'estimation correcte du modèle choisi. Le choix de l'échelle territoriale de l'analyse est également un paramètre qui a une influence forte sur les résultats.

L'étude d'impact économique est devenue un outil standard utilisé par les universités pour faire prendre conscience de l'importance des dépenses dans l'enseignement supérieur les États. Comme les études d'impact économique sont devenues un outil politique, des hypothèses et des méthodes conservatrices devraient être utilisées pour garantir l'objectivité dans le processus de recherche. Etudier les impacts est particulièrement intéressant quand il s'agit de comparer des universités situées dans des régions aux niveaux de développement différents en utilisant le même jeu d'outils tout au long de l'analyse.

L'impact des établissements d'enseignement supérieur sur l'économie locale est large et varié. Au-delà de l'impact économique à court terme, l'enseignement supérieur a un rôle important dans la création de connaissances et est ainsi une source de croissance à long terme. L'université contribue aussi à l'esprit d'entreprise et à toutes sortes d'autres dimensions de créativité comme l'attraction de populations aux profils culturels et modes de vie variés, etc. La créativité n'est pas qu'une affaire de connaissances. Le processus d'estimation de la valeur actuelle des effets économiques de cette accumulation du capital humain n'est pas encore élaboré.

Pour reconnaître le "système des impacts ", dans le deuxième chapitre de l'article, nous présentons un sommaire théorique et des clarifications des termes. Le troisième chapitre est consacré à la méthodologie des estimations (avec un accent sur les méthodes sans utilisation des matrices input-output). Les problèmes généraux sont présentés en s'appuyant sur les exemples des universités de Szeged et de Lorraine au sein du quatrième chapitre. Les résultats empiriques ont été développés selon la même méthodologie et ils sont ainsi comparables. 


\section{- 2 - \\ Les bases théorétiques}

Le rôle des universités a évolué dans le temps. Selon le document du Conseil Supérieur de l'Education (1994), la mission de formation a été définie comme « le phare dans le développement économique local ». WisSEMA (2009) a suggéré qu'il existait trois générations d'universités, mais PAWLOWSKI (2009) parle déjà d'une quatrième génération. Les caractéristiques des générations sont résumées dans le Tableau 1.

Les générations et les missions ( $c f$. les " objectifs" dans le Tableau 1) des universités sont souvent confondues. On considère l'éducation comme la première mission, la recherche comme la deuxième, et l'utilisation locale des connaissances et l'amélioration de la compétitivité comme la troisième mission des universités (ZUTILUKOVICS, 2014). Même si ces éléments aident à définir les différentes générations, une université de deuxième génération qui a connu des transferts de technologie, ne peut être considérée comme une université de troisième génération. En plus, la non-utilisation de la langue nationale ne facilite pas la communication avec les entreprises locales et le transfert de connaissance au public non-scientifique. La définition des missions est également utile pour délimiter l'ensemble des impacts ou la gestion des problèmes spécifiques comme le rôle des hôpitaux universitaires.

\subsection{Les impacts généraux}

FLORAX (1992) et ses adaptations par GARRIDO-YSERTE et GALLO-RIVERA (2010) ont montré que les effets régionaux et locaux de l'université peuvent être observés dans de nombreux domaines en dehors de l'économie ( $c f$. Tableau 2).

L'étude de l'OCDE (2007) examine l'engagement régional de l'enseignement supérieur via la création de savoir, le transfert du savoir et le développement culturel et communautaire. BRENNAN et al. (2006) complètent les aspects du Tableau 2 par une liste d'une vingtaine d'impacts sociaux, culturels et institutionnels dans le cadre du concept d'accessibilité. DuKE (2008) attire l'attention sur la marginalisation d'une partie de la contribution : les composantes sociale, culturelle et environnementale.

Les impacts non-économiques peuvent créer des impacts indirects économiques à long terme qui devraient être analysés pour avoir une image complète. Ces études sont toujours partielles, comme par exemple les impacts économiques à long terme des changements politico-idéologiques des habitants après avoir fait des études supérieures, et qui ne sont pas pondérables.

\subsection{Les impacts économiques}

Pellenbarg (2005) a modifié le tableau de LAmbooy (1996) pour obtenir une liste complète des impacts économiques ( $c f$. Tableau 3 ). Toutefois, cette classification est un mélange des impacts des trois principales missions des universités (l'éducation, 
Balázs Kotosz, Marie-France GAUNARD-ANDERSON, Miklós LuKovics

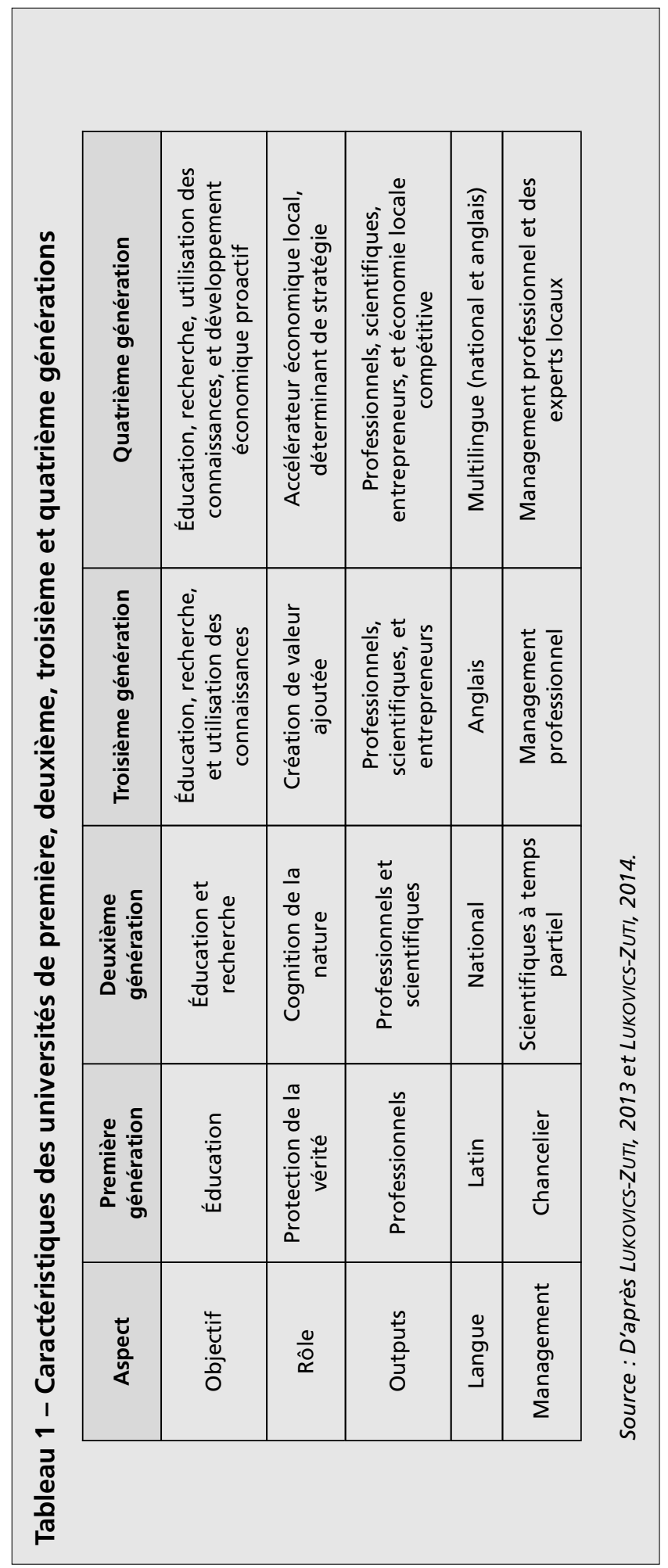




\begin{tabular}{|c|c|}
\hline Impact sur & Exemple \\
\hline Politique & $\begin{array}{c}\text { Changements dans la structure politique, augmentation de la } \\
\text { participation des citoyens, amélioration de l'organisation des processus } \\
\text { politiques }\end{array}$ \\
\hline Démographie & $\begin{array}{c}\text { Impacts sur la croissance de la population, sur la structure de la } \\
\text { population et sur la mobilité }\end{array}$ \\
\hline Économie & $\begin{array}{l}\text { Impacts sur les revenus locaux, la structure industrielle, marché de } \\
\text { l'emploi, la mobilité régionale du travail }\end{array}$ \\
\hline Infrastructure & $\begin{array}{c}\text { Impacts sur le logement, la circulation, les services de santé, commerce } \\
\text { de détail }\end{array}$ \\
\hline Culture & Large offre de biens culturels, influence sur l'environnement culturel \\
\hline Attraction & Influence sur l'image régionale (locale) et l'identité régionale (locale) \\
\hline Éducation & Impact sur le taux de participation, amélioration de la qualité \\
\hline Aspects sociaux & $\begin{array}{l}\text { Impact sur la qualité de vie, influence des étudiants, influence sur } \\
\text { l'image régionale (locale) et l'identité régionale (locale) }\end{array}$ \\
\hline
\end{tabular}

Source : D'après FLORAX (1992) et GARRIDO-YSERTE et GALLO-RIVERA (2010).

la recherche et les relations avec les entreprises); de plus, elle compte en double plusieurs flux (revenus et dépenses de...).

SEGARRA I BLASCO (2004) a divisé les effets « arrière » et « avant » (cf. les Tableaux 4 et 5). Parmi les facteurs de localisation (au lieu d'attractivité), il mentionne également les investissements étrangers et la haute technologie des entreprises qui sont des acteurs typiques de groupes de type technopole. Ces classifications ne sont pas loin du modèle de DuseK (2003) ou LENGYel (2004) qui trient l'impact des intrants et des extrants. DusEK (2003) souligne le rôle de l'origine des finances publiques qui sont les principaux revenus dans le budget de l'université. HuGGins et COOKE (1997) ont transféré les mots-clés aux pilotes (au niveau des intrants) et aux résultats (au niveau des extrants), et dans leur approche, on ne peut pas trouver des mesures concrètes que du côté des pilotes.

Brown et HEANEY (1997) ont conclu que les effets des intrants peuvent être mieux mesurés que les effets des extrants, tandis que la troisième mission des universités, le transfert de connaissances, a des impacts essentiellement sociaux. Ils conseillent de séparer les analyses entre d'une part l'approche économique et, d'autre part, l'approche basée sur les compétences. Malgré tout, BЕCK et al. (1995) soutiennent que les facteurs sociaux (capital humain) doivent être pris en compte, sinon la majeure partie des impacts serait exclue. 


\begin{tabular}{|c|c|}
\hline \multicolumn{1}{|c|}{ Tableau 3 - Impacts économiques régionaux / locaux des universités } \\
\hline Les impacts économiques d'université & Exemple \\
\hline Emploi à l'université & $\begin{array}{c}\text { Nombre d'emplois universitaires et des } \\
\text { institutions connexes }\end{array}$ \\
\hline Dépenses de l'université & $\begin{array}{c}\text { Contributions de l'État, les frais d'inscription, les } \\
\text { bénéfices de l'activité entrepreneuriale, etc. }\end{array}$ \\
\hline $\begin{array}{c}\text { Le revenu et les dépenses des } \\
\text { employés de l'université }\end{array}$ & $\begin{array}{c}\text { Les salaires et traitements, les prélèvements de } \\
\text { la sécurité sociale }\end{array}$ \\
\hline Effets sur le marché du travail & $\begin{array}{c}\text { Effet du travail qualifié sur la productivité, } \\
\text { l'offre de travail flexible des étudiants }\end{array}$ \\
\hline Génération d'entreprise & $\begin{array}{c}\text { Création d'entreprises par les étudiants, les } \\
\text { diplômés et les employés de l'université }\end{array}$ \\
\hline Marketing des connaissances & $\begin{array}{c}\text { La vente de la connaissance dans une variété de } \\
\text { façons : des idées, des cours et des brevets }\end{array}$ \\
\hline
\end{tabular}

Source : PELLENBARG (2005).

Tableau 4 - Impacts régionaux / locaux des universités des intrants

\begin{tabular}{|c|c|}
\hline Acteurs & Changements \\
\hline Ménages & $\begin{array}{c}+ \text { revenu } \\
+ \text { emploi } \\
+ \text { consommation }\end{array}$ \\
\hline Autorités locales & $\begin{array}{c}\text { + base d'impôt } \\
+ \text { services }\end{array}$ \\
\hline Entreprises & + volume d'affaires \\
\hline
\end{tabular}

Source: D’après SEGARRA I BLASCO (2004).

POLÈSE et LÉGER (1979) dans leur ouvrage francophone pionnier, ont détaillé cinq composantes de l'impact : l'impact à court terme sur le niveau régional de production, l'impact sur le stock régional de capital humain, notamment l'impact sur le stock régional des connaissances, l'impact sur l'implantation de nouvelles industries, l'impact sur les aménités urbaines. STOKES et COOMES (1998) ont également souligné l'importance de séparer les effets à court et à long terme, et ont construit une matrice des impacts ( $c f$. Tableau 6 ) dans laquelle la plupart des impacts de POLÈSE et LÉGER est intégrée. 


\begin{tabular}{|c|c|}
\hline Facteurs & Changements \\
\hline Capital humain & $\begin{array}{c}\text { + qualification } \\
+ \text { nouvelles entreprises } \\
\text { + migration }\end{array}$ \\
\hline Connaissance & $\begin{array}{l}\text { + relations université-entreprise } \\
\text { + utilisation extensive des ressources }\end{array}$ \\
\hline Attractivité & $\begin{array}{c}\text { + choix de l'emplacement des ménages et des entreprises } \\
+ \text { possibilités culturelles et sociales }\end{array}$ \\
\hline Entreprises & + recherche et développement, des expositions \\
\hline
\end{tabular}

Source: D'après SEGARRA I BLASCO (2004).

JOHNSON (1994) est le premier qui tient à séparer les impacts locaux et nonlocaux, directs et indirects, mais il insiste également sur les divers impacts négatifs des universités et la nécessité d'une approche nette (avec argumentation que les individus pourraient dépenser plus, si le gouvernement ne les imposait pas pour payer les dépenses des universités). Ces questions interdépendantes seront traitées dans les sous-sections suivantes.

\subsection{Des impacts directs, indirects, primaires, induits, catalytiques}

La classification des impacts est très variée dans la littérature. On peut trouver des classifications en deux, trois ou quatre catégories. Le point commun est la séparation des impacts directs et indirects, où en général (mais pas toujours) les impacts directs incluent les dépenses de l'université, du personnel et des étudiants. Dans une classification plus large, on découvre des impacts induits (KLOPHAUs, 2008), tandis que dans la version à quatre catégories, on peut également trouver des impacts catalytiques (pour ces impacts $c f$. LUKOVICS et DUSEK (2014) pour des institutions de recherche et développement ou DUSEK et LuKOVICs (2011) pour des services commerciaux). La logique de la classification quadruple est présentée en Figure 1.

Malgré les publications sur le sujet depuis quarante-cinq ans, la terminologie n'est pas encore stabilisée, les points de vue de la chaine de valeur sont largement divergents. Le mélange contradictoire des définitions des impacts peut être bien représenté par la juxtaposition ( $c f$. Tableau 7) de ceux de GARRIDO-YSERTE et GALLORIVERA (2010) et de l'école française représentée par GAGNOL et HÉRAUD (2001) et BASLÉ et LE BOULCH (1999). 
Balázs Kotosz, Marie-France GAUnARD-ANDERson, Miklós LuKovics

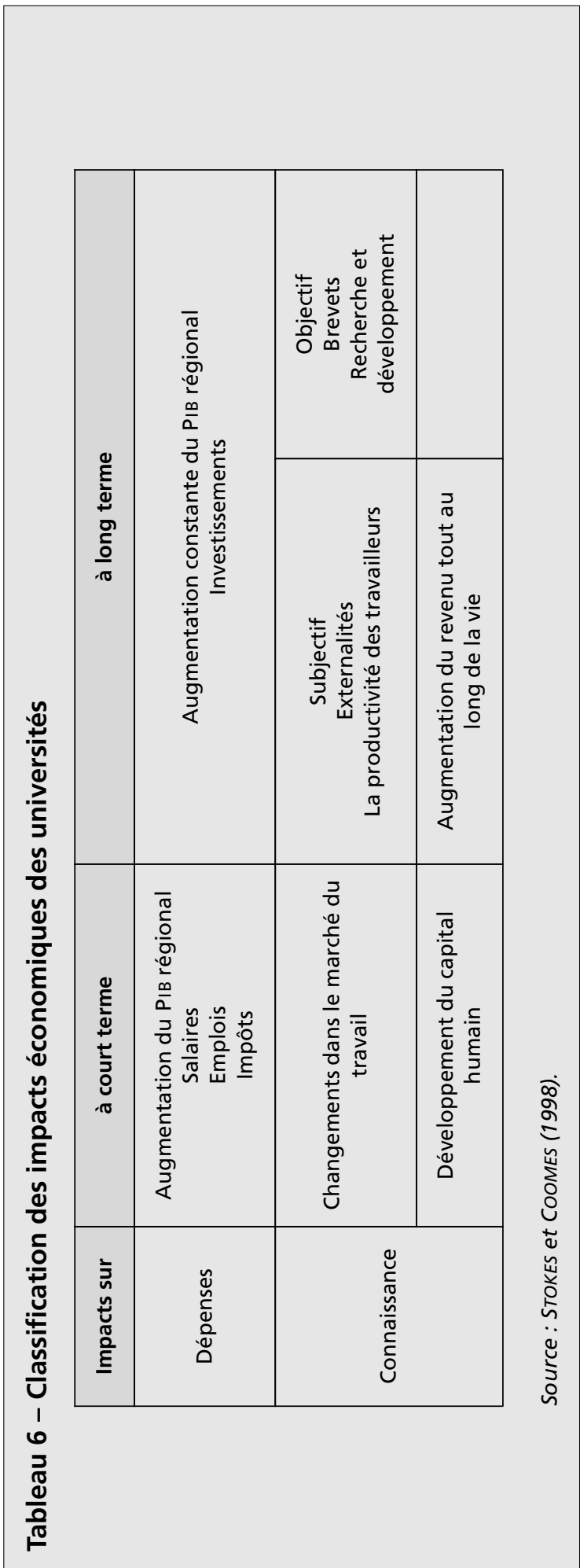


Figure 1 - Impacts directs, indirects, induits et catalytique

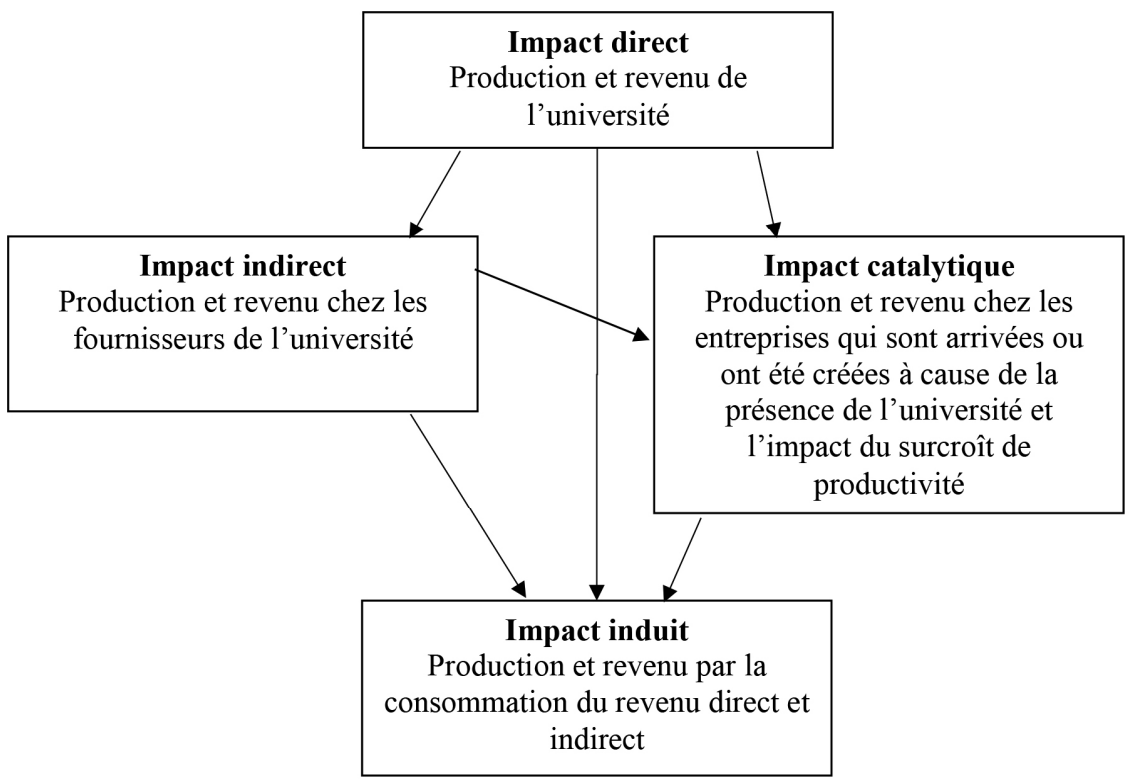

SOurCe : AUTEURS.

\section{Tableau 7 - Classification des impacts régionaux des universités}

\begin{tabular}{|c|c|c|}
\hline \multirow{2}{*}{ Impact } & \multicolumn{2}{|c|}{ Interprétation } \\
\hline & GARRIDO-YSERTE et GALLO-RIVERA & GAGNOL et HÉRAUd \\
\hline Direct & $\begin{array}{l}\text { Dépenses locales de l'université, de ses } \\
\text { employés et de ses étudiants }\end{array}$ & $\begin{array}{l}\text { Consommation de l'établissement } \\
\text { d'enseignement et sa capacité à } \\
\text { attirer des employés et des } \\
\text { étudiants }\end{array}$ \\
\hline Indirect & $\begin{array}{l}\text { Revenu multiplié, les transactions } \\
\text { générées par la consommation locale } \\
\text { chez des entreprises locales qui ne sont } \\
\text { pas directement rattachées à l'université }\end{array}$ & $\begin{array}{l}\text { Qualification de la main-d'œuvre } \\
\text { locale, recherche et } \\
\text { développement industriel, pôle } \\
\text { d'attraction pour des entreprises }\end{array}$ \\
\hline Induit & $\begin{array}{l}\text { Les dépenses des visiteurs, les effets sur } \\
\text { les institutions financières, sur la valeur } \\
\text { des immobiliers, sur le choix résidentiel } \\
\text { et la localisation des entreprises, etc. }\end{array}$ & Effet multiplicateur \\
\hline
\end{tabular}

Source : GARRIDO-YSERTE et GALLO-RIVERA (2010) et GAGNOL et HÉRAUD (2001). 
Dans cette confusion, nous recommandons d'utiliser l'expression "impact induit » à tous les effets qui sont générés par le processus de multiplication keynésienne. Dans les classifications, la séparation des impacts directs et indirects est souvent artificielle. Nous séparons les dépenses personnelles de l'achat des actifs et des investissements dans le but de séparer le local et le non-local, mais ni le revenu des employés, ni les dépenses sur des investissements ne sont dépensés nécessairement sur place. Le terme de DusEK et Kovács (2011) des « effets primaires » nous semble plus pertinent, parce que les étudiants sont des parties intégrantes de l'université. L'impact catalytique de LUKOVICS et DUSEK, l'impact indirect de GAGNOL et Héraud et l'impact induit de GARRIDO-YSERTE et GALLO-RIVERA ont presque le même contenu. Alors qu'elle est peu répandue dans la littérature, l'expression catalytique décrit mieux le contenu de cette catégorie que "indirecte " ou " induite " (induite est la moins adaptée). Nous proposons l'utilisation des termes " primaire», "induit " et " catalytique ».

\subsection{Des effets nets}

L'idée de JOHNSON (1994) de considérer une approche nette a entraîné des débats dans les articles y référant. Généralement, l'impact brut est plus facile à définir et à calculer; le nombre de points de vue à appliquer dans l'approche nette est de plus en plus large. JOHNSON considère que les individus dépenseraient plus, si le gouvernement ne les taxe pas pour payer les dépenses des universités. Même cette question en soulève une autre, celle du calcul de la proportion de taxes collectées dans la région où l'université est présente. BECK et al. (1995) donnent une description détaillée des transformations nécessaires des subventions gouvernementales et des impôts afin d'être correctement pris en compte.

MARTIN (1998) identifie et évalue les effets des dépenses de subsistance des étudiants locaux, la différence de leurs dépenses comme étudiants et travailleurs, et les impacts des subventions gouvernementales. Ces dernières doivent être gérées avec prudence parce que les subventions qui viennent du niveau national (fédéral dans le cas de l'exemple du Québec) doivent être réparties selon leur échelle territoriale. Si l'on réfléchit en termes de contre factuel et d'effet l'éviction, toute autre dépense publique que celle affectée à l'université aurait aussi un effet multiplicateur, probablement du même niveau.

BRENNAN et al. (2006) accentuent les externalités négatives des étudiants (leur demande déforme le marché local des services).

SIEGFRIED et al. (2007) posent plusieurs questions qui nécessitent des réponses : - Si l'université n'existait pas, où le personnel travaillerait ? Les enseignantschercheurs sont-ils différents du personnel administratif ?

- Si l'université n'existait pas, qu'est-ce que le personnel ferait ?

- Si l'université n'existait pas, est-ce que les étudiants poursuivraient des études ailleurs ou travailleraient-il localement?

- Quelle est la différence du niveau des connaissances locales avec et sans l'université?

- Quelle serait la différence des prix de l'immobilier? 
Il ne faut pas oublier que ces questions sont également liées au choix de l'échelle territoriale. La mobilité des étudiants peut être connue par enquête et les conséquences sont remarquablement différentes si l'université est seule dans la région ou est une des universités d'une grande métropole. Le niveau des connaissances locales est traduit en euros par la différence des salaires, mais le niveau de prix de l'immobilier ne peut être comparé qu'avec des villes de référence où il n'y a pas d'enseignement supérieur.

\section{- 3 - \\ Méthodologie des estimations}

\subsection{Les familles de méthodes}

Dans leur récent sommaire méthodologique, GARRIDO-YSERTE et GALLO-RIVERA (2010) divisent les méthodes d'estimations en deux groupes : directes et indirectes, selon les sources d'informations. Les méthodes directes utilisent des données primaires, les indirectes sont calculées par des données secondaires. À notre avis, l'utilisation de l'une ou l'autre des méthodes s'expliquera davantage en fonction des matrices input-output disponibles.

\subsubsection{Les méthodes en présence de matrice input-output}

Une matrice détaillée d'input-output de l'économie régionale facilite le calcul en utilisant des informations secondaires sur les liaisons économiques des agents intra et extrarégionaux. Si la structure sectorielle des dépenses de l'université est connue, les impacts primaires et induits peuvent être estimés sans difficulté (cf. REHÁK et al., 2015 pour les formules), mais avec une modélisation économétrique, il est possible de disposer facilement des impacts catalytiques liés au changement de la fonction de production (WEST et JACKSON, 1998). Pour les impacts primaires et induits, quelques opérations matricielles sont suffisantes, si la structure des dépenses de l'université est mise en conformité avec la structure de la matrice. Il faut souligner que la plupart des territoires régionaux et locaux ne disposent pas de matrice input-output et la création d'une telle matrice soulève plus de problèmes que de bénéfices.

MARTIN (1998) sépare les méthodes statiques et dynamiques, en prenant en compte les changements de la matrice input-output suscitée par l'activité de l'université (accumulation des connaissances, etc.)

\subsubsection{Les méthodes sans matrice input-output}

Le premier ouvrage sur le sujet (CAFFREY et ISAACS, 1971) a proposé une méthode directe comme réponse à la demande d'American Council of Education (ACE). Leur méthode permet d'estimer les impacts sur les entreprises locales, sur les individus locaux et sur l'administration locale. En raison de sa complexité, plusieurs simplifications ont été proposées (BLEANEY et al., 1992 ; HUGGINS et COOKE, 1997 ; LESLIE et LEWIS, 2001). Les études américaines, où l'échelle territoriale est au niveau d'un état fédéral, préfèrent cette méthode. 
La solution de RYAN (RYAN et MALGIERI, 1992) est la simplification de la méthode ACE par le remplacement de données primaires par des données secondaires : au lieu de réaliser des sondages parmi les étudiants, les enseignants-chercheurs et le personnel administratif, ils suggèrent d'utiliser des statistiques régionales. Si le comportement des employés et des étudiants de l'université est proche de la moyenne régionale, cette solution peut diminuer la quantité de travail nécessaire sans perte significative de l'information.

\subsection{Méthodologie conseillée pour des analyses au niveau local}

Quand l'analyse est réalisée au niveau local (impact sur la ville ou l'aire de la communauté urbaine), il est impossible d'utiliser des matrices input-output qui correspondent à ce niveau territorial. Certaines études (par exemple BIGGAR ECONOMICS, 2015) ont choisi le niveau territorial en fonction des données accessibles, mais une approche scientifique (surtout spatiale) ne peut pas négliger les différences d'échelle et ne doit pas utiliser des moyennes européennes calculées au niveau local.

En principe, les versions simplifiées de la méthode ACE utilisent un multiplicateur keynésien régional. Dans l'article de BlEANEY et al. (1992) ou HugGins et COOKE (1997), nous pouvons trouver une déduction mathématique de la formule du multiplicateur keynésien régional. Cette méthode est la plus utilisée pour le calcul dans les études de cas européens, avec une série d'inconvénients et de déficiences. Sa simplicité fait qu'elle est très utilisée, et une échelle relativement fine de données est nécessaire.

SIEGFRIED et al. (2007) montrent que les multiplicateurs régionaux ont une médiane de 1,7; ceux qui sont plus grands que 2 sont suspects. HeRMANNSSON et al. (2013) analysent l'impact de l'échelle territoriale sur les multiplicateurs : le choix du niveau local amplifie la multiplication des contributions locales des individus (volume d'impôts) ; alors le financement extérieur (non local) crée des multiplicateurs énormes des dépenses publiques locales.

Pour les établissements dans des régions (ou villes) où une matrice input-output régionale n'est pas accessible, nous recommandons l'application d'une méthode mixte : avec les principes du modèle de HugGins et COOKE (1997) et BLACKWELL et al. (2002), mais en appliquant les conseils de RYAN sur l'utilisation des données secondaires quand les données primaires ne sont pas accessibles, mais les données régionales sont fiables. L'enquête est inévitable dans le cas des dépenses locales des étudiants et fortement conseillée pour l'estimation des dépenses locales des employés. Le niveau local induit un problème additionnel : les revenus et les dépenses de l'université doivent être distribués par des clés forfaitaires où la source n'est pas évidente.

Une application de la méthode conseillée est présentée dans le chapitre suivant par les exemples de l'Université de Szeged (Hongrie) et de l'Université de Lorraine (France). Les résultats de ces deux études de cas sont parfaitement comparables à cause de la méthodologie identique. Le modèle du multiplicateur keynésien a été 
modifié aux points suivants : (1) nous utilisons des habitudes de consommation locales (et la propension marginale locale à consommer), (2) nous calculons la production primaire et l'effet de la consommation en deux étapes. Par ce modèle, la multiplication est la fonction des facteurs suivants :

- Taux d'impôt sur le revenu des particuliers (taux moyen) $(t)$

- Taxe sur la valeur ajoutée (taux moyen) $(n)$

- La propension marginale à consommer à l'échelle locale $(c)$

- Proportion de la consommation locale des étudiants $(d)$

- Proportion de la consommation locale des employés $(e)$

- Proportion de la consommation locale de l'université $(b)$

- Proportion de la consommation de l'économie locale $(f)$

Par le calcul en deux étapes, la formule du multiplicateur se résume à :

$$
\frac{1}{1-f \cdot c \cdot(1-t) \cdot(1-n)}
$$

Le calcul de l'impact primaire s'est opéré en deux tours. À partir de l'étape 4 du Tableau 8, les nouveaux tours sont celui du processus de multiplication classique (avec le multiplicateur régional montré ci-dessus).

\section{Tableau 8 - Les étapes du calcul}

\begin{tabular}{|c|c|c|}
\hline Étape & Libellé & Formule \\
\hline 1. & Effet de production de premier tour & $O_{1}=P+A+b \cdot V$ \\
\hline 2. & Effet de revenu de premier tour & $I_{1}=(1-t) \cdot\left(O_{1}-b \cdot n \cdot V\right)$ \\
\hline 3. & Effet de production deuxième tour & $O_{2}=d \cdot S+e \cdot c \cdot I_{1}+M$ \\
\hline 4. & Effet de revenu deuxième tour & $I_{2}=(1-t) \cdot(1-n) \cdot O_{2}$ \\
\hline 5. & Effet de production troisième tour & $O_{3}=e \cdot c \cdot(1-t) \cdot(1-n) \cdot O_{2}$ \\
\hline 6. & Effet de revenu troisième tour & $I_{3}=(1-t) \cdot(1-n) \cdot O_{3}$ \\
\hline
\end{tabular}

Source : AUTEURS.

Comme l'origine d'une part des données est l'enquête ou l'estimation, des analyses de sensibilités sont fortement conseillées. 


\section{-4 - \\ Résultats empiriques à Metz et à Szeged}

\subsection{Les sources d'information et le calcul}

Metz est située dans la région Lorraine en France avec une population d'environ 130000 personnes (288 000 pour l'unité urbaine). L'université de Metz fut fondée en 1970 ; l'Université de Lorraine est le résultat d'une fusion des universités dans la région (Nancy I, Nancy II, Metz et plusieurs petits établissements) et elle est la deuxième plus grande université en France par le nombre d'étudiants. Toutefois, notre analyse concerne uniquement la ville de Metz. Sur les 50000 étudiants de l'université, environ 13000 peuvent être localisés sur les campus de Metz répartis en six facultés. La zone d'attraction des campus de Metz est plutôt régionale. Les résultats des estimations peuvent être trouvés dans la Figure 2.

Szeged est le centre de la région Plaine du Sud avec une population de 160000 personnes (unité urbaine 200 000). L'université fut fondée en 1872 ; les résultats scientifiques d'un chercheur (Albert Szent-Györgyi) ont été reconnus en 1937 par le prix Nobel. Depuis 2000, après la fusion des établissements à Szeged et dans le département de Csongrád, elle recouvre 12 facultés avec 23000 étudiants. L'université intègre aussi un centre médical (clinique de recherche). Les résultats de nos estimations peuvent être trouvés dans la Figure 3.

En raison du rôle de Metz dans l'Université de Lorraine et des sources possibles de données primaires (enquêtes auprès des étudiants), le seul choix de niveau territorial était le niveau local.

Les données de base du calcul des deux établissements se trouvent dans le Tableau 9.

Les données sur les dépenses de l'université sont accessibles à partir des sources d'information semi-publiques (budget de l'université) ${ }^{2}$. Pour trouver la valeur de proportion de consommation locale de l'université ( 0,7 en Hongrie et 0,8 en France), une analyse détaillée du budget était nécessaire. Le niveau local pose des problèmes d'interprétation dans le cas des achats (par exemple l'énergie), mais si le fournisseur direct est local, la littérature les considère comme locaux (SZÉKELY, 2013).

Nous supposons que les employés disposent d'un revenu supplémentaire de $20 \%$ sur leur salaire à l'université (éditions, consultation, bourses de recherche, etc.). Sans estimations locales disponibles, une valeur fréquemment utilisée a été définie. Afin de pouvoir comparer correctement les deux cas français et hongrois, nous n'avons pas tenu compte des sommes payées dans le cadre des retraites, parce que les employeurs en Hongrie ne payent pas de retraites directement. En l'absence d'enquêtes auprès des employés, la part de leur consommation locale $(0,75$ en Hongrie et 0,6 en France) a été estimée en considérant leur lieu de résidence. 


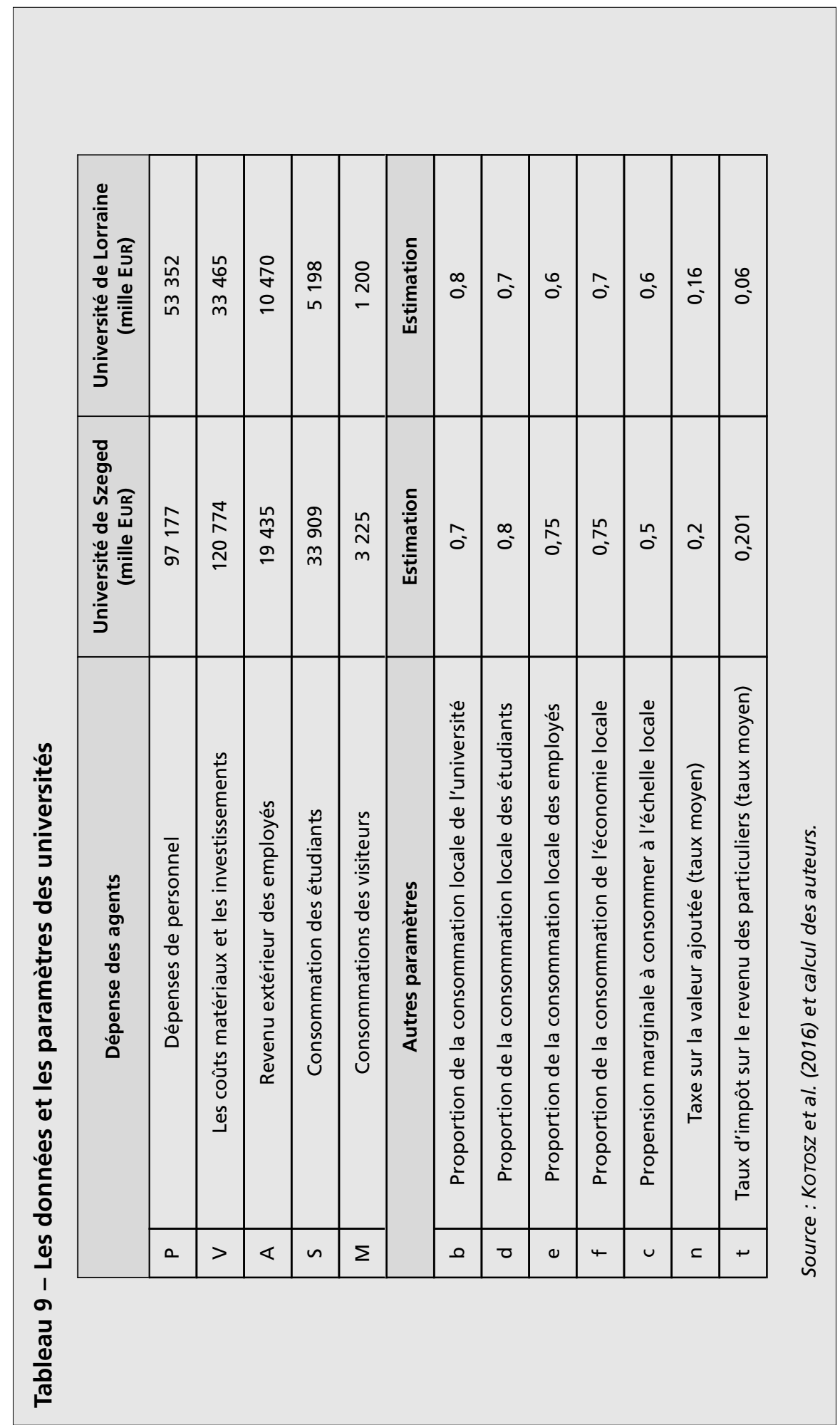


La part de la consommation dans l'économie locale $(0,75$ en Hongrie et 0,7 en France) est la moyenne de celle des acteurs analysés. Le décalage entre les deux cas étudiés reflète les différences de l'importance des villes au sein de leurs espaces agglomérés (l'aire urbaine de Metz a plus de deux fois plus d'habitants que la commune, mais l'aire urbaine de Szeged en dehors de la ville est moins peuplée).

Pour cartographier les dépenses des étudiants, nous leur avons demandé de remplir un questionnaire de quatre pages avec de nombreuses questions de détails pour pouvoir vérifier les données générales. Ainsi la donnée des dépenses des étudiants a été basée sur un échantillon, nous avons multiplié la moyenne de l'échantillon par le nombre d'étudiants inscrits à l'université. Dans le cas des étudiants à temps partiel (qui n'ont pas été questionnés dans l'enquête), nous avons compté un cinquième de la consommation locale des étudiants à temps plein. La plupart de leur consommation serait réalisée si l'université n'existait pas. Le taux d'échantillonnage (au niveau des questionnaires utilisables c'est-à-dire bien remplis sans contradictions importantes) était légèrement supérieur à $3 \%$. Les différences éventuelles entre nos résultats et ceux de sondages nationaux peuvent être expliquées par la composition des étudiants selon leur origine géographique. Leur part de la consommation locale $(0,8$ à Szeged et 0,7 à Metz) est entièrement fondée sur les enquêtes.

L'estimation des dépenses des visiteurs (3 millions d'euros à Szeged et 1 million d'euros à Metz) est calculée en prenant en compte les colloques et les événements de remise de diplômes.

Pour estimer la fonction de consommation à l'échelle locale, nous pouvons suivre deux voies différentes. D'une part, nous pouvons utiliser les statistiques nationales ou régionales. Selon les études, en Hongrie, les fonctions locales et nationales ne sont pas significativement différentes (ÁRVAY et MENCZEL, 2001 ; VIDOR, 2005). D'autre part, les sondages locaux peuvent également servir de point de départ. Les résultats généraux nationaux pour la Hongrie sont autour de 0,6; les résultats régionaux en France entre 0,45 et 0,7. Les sondages locaux des étudiants montrent une propension marginale à consommer inférieur à 0,5 en Hongrie et autour de 0,5 en France. Les valeurs utilisées dans le calcul (0,5 en Hongrie, 0,6 en France) ont été calibrées d'une manière conforme à ces estimations.

Pour les taux moyens d'impôt (recettes d'impôts / revenu imposable), nous avons utilisé les estimations de la Banque Nationale de Hongrie, de l'INSEE et du Ministère des finances et des comptes publics en France, et les taux régionaux lorsqu'ils étaient accessibles.

\subsection{Analyse comparative des résultats}

Les résultats peuvent être comparés aux exemples internationaux, et aux références nationales. Depuis l'article de CAFFrEY et IsAACS (1971), une centaine d'études scientifiques et plus d'études commerciales ont été publiées. Pour la Hongrie, deux études de cas à Győr (DUSEK, 2003 ; DuSEK et KovÁCS, 2011) et deux études de cas des écoles de commerce (KOTOSZ, 2013) sont connues. Certaines approches 
ont également été faites à Pécs (MEZEI, 2005), mais cette recherche n'a pas permis d'atteindre un résultat quantifié. En France, les résultats de Strasbourg (GAGNOL et HÉRAUD, 2001), Rennes (BASLÉ et LE BOULCH, 1999) et de l'Université du Littoral (MILlE, 2004) ont été publiés dans des revues, mais les analyses d'Orléans-Tours, Saint-Étienne et Reims (Lu et PERRON, 2013) restent sous forme de document de travail.

L'Université de Szeged est deux fois plus importante que l'Université de Lorraine à Metz (environ deux fois plus d'enseignants, d'étudiants, de facultés, etc.). L'indicateur du nombre relatif d'étudiants $(1,77)$ qui détermine les résultats, marque bien cette différence, qui apparaît également à travers les revenus (Szeged : 230 millions d'euros et Metz 123 millions d'euros), les dépenses et l'impact des universités (l'impact sur revenu local : 219 millions d'euros et 119 millions d'euros). La structure des dépenses des universités est bien différente. Les dépenses de l'Université de Lorraine sont lourdement dominées par le coût des employés ; indépendamment du coût qu'aurait un gros investissement ponctuel, ils représentent environ $90 \%$ des dépenses. En Hongrie, les dépenses de personnel restent inférieures à 50 \%. Les bourses payées aux étudiants sont également beaucoup plus importantes en Hongrie ( 9 millions d'euros contre 1 million d'euros).

Les taux d'impôts plus élevés et la propension marginale à consommer plus bas en Hongrie ont mené le multiplicateur régional à une valeur inférieure à celui calculé en France.

L'impact total au niveau des revenus est de 219 millions d'euros à Szeged et de 119 millions d'euros à Metz (Tableau 10), soit presque les mêmes sommes que les revenus des universités (respectivement 230 millions d'euros et 123 millions d'euros).

\section{Tableau 10 - Les impacts économiques à Szeged et à Metz}

\begin{tabular}{|c|c|c|}
\hline Impacts & $\begin{array}{c}\text { Université de } \\
\text { Szeged } \\
\text { (million EUR) }\end{array}$ & $\begin{array}{c}\text { Université de } \\
\text { Lorraine } \\
\text { (million EuR) }\end{array}$ \\
\hline Impact primaire (revenu) & 167 & 80 \\
\hline Impact induit (revenu) & 52 & 39 \\
\hline Impact total (revenu) & 219 & 119 \\
\hline Impact primaire (production) & 240 & 94 \\
\hline Impact induit (production) & 74 & 46 \\
\hline Impact total (production) & 314 & 140 \\
\hline
\end{tabular}


Il est possible de faire une comparaison au niveau national à partir de l'impact total par étudiant. Dans Kotosz et al. (2015), selon les études de cas américains, l'impact par étudiant varie entre 15000 et 50000 euros, résultats obtenus par la méthode input-output. En Europe occidentale, il ne monte qu'à hauteur de 10000 à 20000 euros (mais seulement - strictement net - 4000 euros à Strasbourg ${ }^{3}$ selon GAGNOL et HÉrAUD, 2002) ; toutefois en Europe orientale, il est au-dessous de 10000 euros. Étant donné les circonstances, les valeurs autour de 11000 euros dans les deux villes analysées semblent tout à fait normales parce qu'en Hongrie, l'hôpital universitaire renforce l'impact (sans augmenter le nombre des étudiants).

L'autre voie de comparaison est l'impact (comme valeur ajoutée à l'économie locale ou régionale) en pourcentage du PIB régional. Les cas américains indiquent plutôt un rapport entre 0,1 et 3,0 \% ; en revanche, en Europe, celui-ci reste inférieur à $0,1 \%$. Les deux villes comparées sont aux seuils inférieur et supérieur des références, avec le taux de $4 \%$ à Szeged et de 0,02 \% à Metz. Cette magnitude de différence est expliquée par plusieurs facteurs :

- À l'Université de Szeged, il y a un hôpital universitaire, qui augmente significativement l'impact.

- L'Université de Szeged se trouve dans une région qui est parmi les quinze plus pauvres régions de l'Union Européenne (PIB par habitant).

- L'Université de Lorraine a des campus plus importants qu'à Metz, alors pour une comparaison au niveau régional, toute l'université devrait être prise en compte.

Dans cet article, avec un objectif plutôt méthodologique, les résultats de l'analyse de sensibilité ne sont pas affichés, mais ils suggèrent une incertitude de 15-20 \% des estimations. Même avec cette incertitude, les impacts ne sont pas significativement plus importants que les dépenses publiques (y compris régionales et locales) sur les universités, alors les multiplicateurs de 5 à 10 ne sont valables que dans les établissements où les frais d'inscription sont au niveau des coûts réels des études.

\subsection{Les impacts hors de portée de notre analyse}

Selon la méthodologie recommandée, les impacts primaires et induits, soit les impacts du côté des inputs et partiellement du côté des outputs (attraction des visiteurs, valeur directe des contrats de recherche) peuvent être estimés. La démarche empirique suggère un multiplicateur local entre 1,3 et 1,5 sur les impacts primaires, mais l'impact total sur le revenu local est presque le même que les revenus de l'université.

Selon les modélisations, les impacts catalytiques ont au moins la même importance que les primaires et induits, mais VARGA (2001) enregistre un effet d'agglomération très important dans le transfert de connaissance. Par conséquent, dans le cas des petites écoles supérieures, les effets induits de la création de connaissance sont inférieurs aux effets économiques à court terme, mais les grandes universités peuvent avoir un impact deux à cinq fois plus important du côté des extrants (MARTIN, 1998) Ces résultats montrent que les impacts catalytiques forment la vraie valeur ajoutée des universités. 
Il existe de nombreuses méthodes pour estimer les impacts des inputs, alors qu'il n'y a pas de méthodologie cohérente pour mesurer ceux des outputs. La cause de ce manque méthodologique est complexe :

- Les impacts catalytiques sont multiples, il n'est pas possible de créer une méthode de « one size fits all ».

- Ces impacts sont à long terme et il faudrait un grand décalage temporel et une enquête spécifique de type quasi historique pour les évaluer.

- Il y a des enseignants-chercheurs et des étudiants dans toutes les universités, mais en plus certaines d'entre elles possèdent un parc scientifique, un incubateur ; les possibilités de recherches débouchant sur des brevets scientifiques sont différentes selon chaque domaine scientifique.

Les idées de BIGGAR (2015) ou BONNET et al. (2014) suggèrent deux points de vue possibles : soit des estimations sommaires (basées sur des données européennes ou nationales) sans tenir compte des spécialités locales, soit des enquêtes précises et des données détaillées de l'université, mais ces données sont gérées comme des informations confidentielles et sont en général inaccessibles.

Les positions de la science sont au stade de la collecte des indicateurs qui décrivent bien les activités de la troisième mission de l'université (JONGBLOED, 2008 ; RITSILA et al., 2008 ; KотоsZ et al., 2015), mais les méthodes pour convertir ces indicateurs de types d'activités (connaissance des activités présentes sans que soient révélées leurs résultats) au niveau de l'impact en euros ne sont pas encore élaborées. L'étude de Cross et Pickering (2008) confirme ce phénomène dans le cas de la valeur culturelle. Le projet FP7 "EvaRIO ${ }^{4}$ a fait une avancée importante dans ce domaine. Les chercheurs ont utilisé des outils avancés, comme analyse des réseaux sociaux. 


\section{- 5 - \\ Conclusion}

Dans notre article, nous présentons un aperçu des méthodes d'évaluation des impacts locaux ou régionaux des universités; nous analysons les désaccords de la littérature et nous formulons des conseils pour des analyses au niveau local où des matrices input-output ne sont pas accessibles. Nous avons également résumé les résultats d'une étude comparative de deux universités, une en Hongrie et une en France.

Il faut tout d'abord constater que les résultats sont très sensibles à la méthode générale appliquée. Un modèle d'économie régionale peut recouvrir des impacts dynamiques comme les conséquences des changements de la productivité globale des facteurs. Puis, après avoir choisi la méthode, l'analyse effectuée a apporté une meilleure connaissance au niveau de la collecte des données et au niveau des effets de la qualité des données sur les résultats.

L'acquisition des données nécessaires n'était pas évidente. Le budget de l'université est semi-public et n'est pas accessible en dehors du système informatique de l'université. Les données détaillées, comme le nombre et la valeur des contrats de recherche avec des entreprises, sont considérées comme informations sensibles et elles ne peuvent pas être obtenues.

La consommation des étudiants ne peut être connue que par des enquêtes. Les enquêtes en ligne atteignent la majorité des étudiants, mais ne sont pas efficaces. Selon notre expérience, le taux de réponse est inférieur à 0,1\%. Les enquêtes personnelles sont beaucoup plus efficaces, mais il faut compter au moins six semaines pour les réaliser (deux à trois semaines de préparation suivies par trois à quatre semaines d'exécution), voire plus dans le cas des universités multi-campus. De même, les étudiants ont une notion de l'argent très limitée; la majorité d'entre eux y a été confrontée pour la première fois lors de la récapitulation de leurs dépenses mensuelles dans le cadre de l'enquête. Par conséquent, ils sous-estiment les coûts de la vie ; l'idée préconçue - suggérée par les employés des universités - que les étudiants de certaines facultés (par exemple économie ou droit) sont d'origines sociales plus aisées, n'a pas été prouvée par les enquêtes.

Une enquête auprès des employés était pratiquement impossible à mener à cause des droits et des libertés de la personne. Le point le plus important, la masse de salaire, est visible dans le budget; de ce fait, la part de la consommation locale est la seule donnée manquante. La comparaison internationale a fait ressortir des problèmes spécifiques : des systèmes de retraite diversifiés, des systèmes d'impositions et de cotisations sociales différentes. Comment peut-on calculer la consommation des retraités de l'université ? Sont-ils toujours présents sur le territoire ? Comment estil possible de comparer la consommation générée par les systèmes d'assurance maladie ? La réponse ne pouvait être que partielle en considérant les éléments communs aux deux pays. 
La structure scientifique de l'université est également un facteur déterminant de l'impact. Les facultés des sciences médicales (y compris dentaires et pharmaceutiques) possèdent souvent un hôpital universitaire dont le budget peut être inclus dans le budget de l'université (cf. le cas de Szeged), ce qui augmente remarquablement l'impact. Les facultés d'ingénieur ont plus de contrats de recherche appliquée que les facultés des lettres, alors le nombre des IuT rehausse l'impact par étudiant.

Globalement, on peut constater que l'exactitude des estimations dépend des impacts et des circonstances pris en compte, les erreurs de calcul et des données du modèle choisi sont moins décisives. La difficulté à mesurer l'impact le plus intéressant qui est l'impact catalytique reste sans percée révolutionnaire.

\section{Remerciements}

Le premier auteur est reconnaissant au projet Campus Hungary dans le cadre du programme TÁMOP-4.2.4B/2-11/1-2012-0001 pour la possibilité de recherche en France, et le soutien financier du programme TÁMOP-4.2.1.D-15/1/KONV-20150002 "Tudás-ipar igényeit kiszolgáló felsőoktatási szolgáltatások megalapozása a Dél-Alföldi régióban" pour la recherche en Hongrie. 
Balázs Kotosz, Marie-France GAUnARD-Anderson, Miklós LUKOVICS

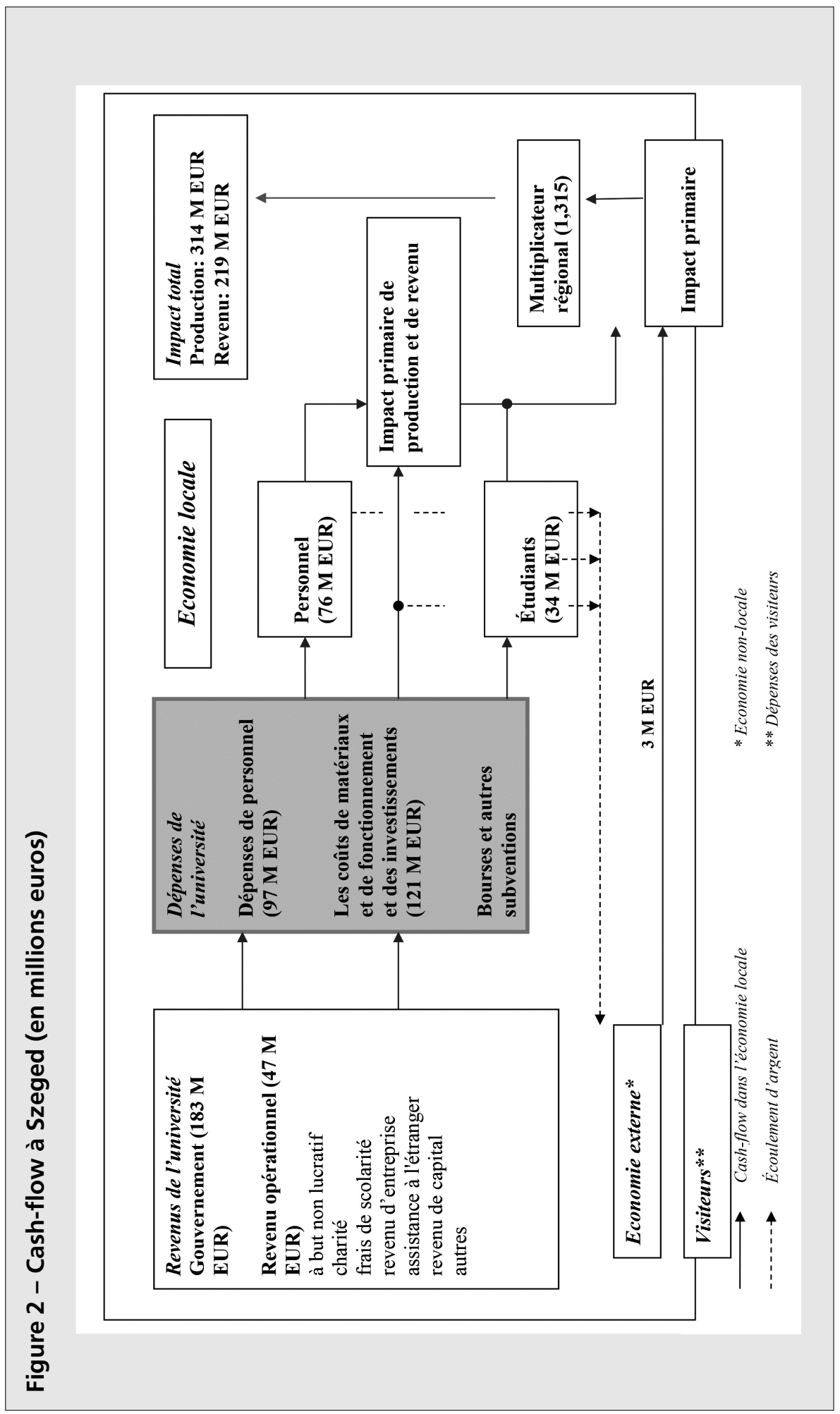




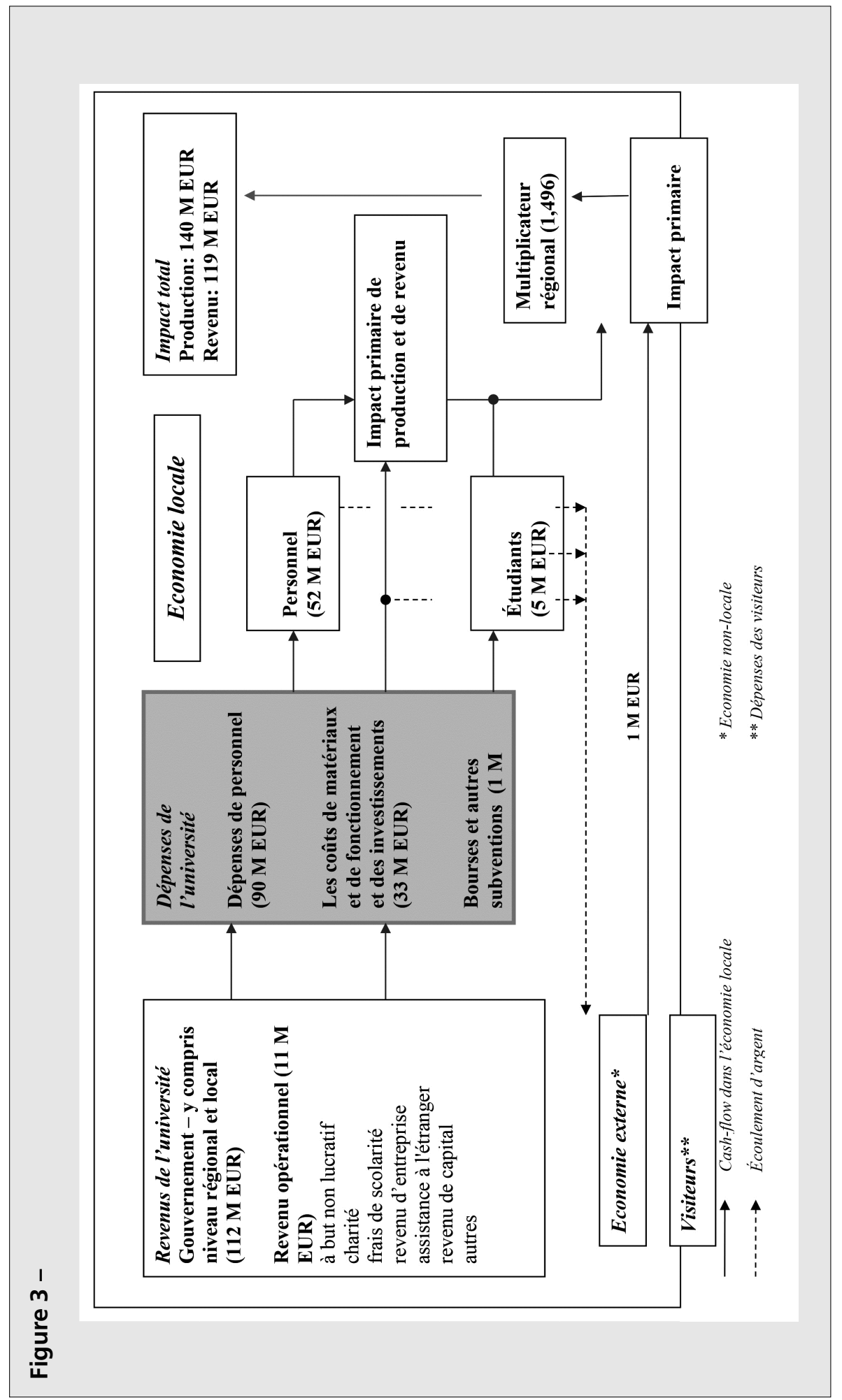




\section{Références bibliographiques}

alves J, Carvalho L, Carvalho R, Correia F, Cunha J, Farinha L, Fernandes J, Ferreira M, lucas E, mourato J, Nicolau a, Nunes S, Nunes S, Oliveira P, Pereira C, Pinto S, Silva J (2015) The impact of polytechnic institutes on the local economy. Tertiary Education and Management 12(2): 1-18.

ÁRVAY Z-S, MENCZEL P (2001) A magyar háztartások megtakarításai 1995 és 2000 között. Közgazdasági Szemle 48: 93-113.

BASLÉ M, LE BOULCH J-L (1999) L'impact économique de l'enseignement supérieur et de la recherche publique sur une agglomération de Rennes. Revue d'Économie Régionale et Urbaine 1999-1: $115-134$.

BeCk R, Elliott D, Meisel J, Wagner M (1995) Economic impact studies of regional public colleges and universities. Growth and Change: 245-260.

BIGgar ECONOMICs (2015) Economic Contribution of the LeRU Universities. Biggar Economics, Roslin. Téléchargé le 20 septembre 2015 [en ligne] http://www.leru.org/files/publications/LERU_Economic_ Contribution_-_Report.pdf

BlaCKWELL M, COBB S, WeinBerg D (2002) The Economic Impact of Educational Institutions: Issues and Methodology. Economic Development Quarterly 16(1): 88-95.

Bleaney M-F, Binks M-R, Greenaway D, Reed G, Whynes D-K (1992) What does a university add to its local economy? Applied Economics 24: 305-311.

Bonnet J, De Lema D-G-P, MARTinez-AbarCA-PAStor C, Rizzo U (2014) Les déterminants de la création de spin-off académiques. Une comparaison internationale dans les établissements supérieurs de Caen, Carthagène et Ferrare. CREM Working Paper, WP 2014-17.

BRENNAN J, LITTLE B, LOCKE W (2006) Higher education's effects on disadvantaged groups and communities. Centre for Higher Education Research and Information, London.

BROWN K-H, HEANEY M-T (1997) A Note on Measuring the Economic Impact of Institutions of Higher Education. Research in Higher Education 38(2): 229-240.

CAFFREY J, ISAACS H-H (1971) Estimating the impact of a College or University on the Local Economy. American Council on Education, Washington, DC.

CONSEIL SUPÉRIEUR DE L'EDUCATION (1994) L'enseignement supérieur et le développement économique. Conseil Supérieur de l'Education, Québec.

Cross E, PICKERING H (2008) Université provinciale de Laponie : collaborer pour promouvoir le développement régional. Politiques et gestion de l'enseignement supérieur 20 (2) : 135-146.

DUKE (2008) Engagement régional des universités : éviter la confusion, malgré d'inéluctables contradictions. Politiques et gestion de l'enseignement supérieur 20 (2) : 99-114.

DUSEK T (2003) A felsőoktatás lokális termelésre és jövedelmekre gyakorolt hatása. In: RECHNITZER J, HARDI T (eds) A Széchenyi István Egyetem hatása a régió fejlödésére. Széchenyi István Egyetem Gazdaságés Társadalomtudományi Intézet, Györ : 60-71.

DUSEK T, KOVÁCS N (2011) A Széchenyi István Egyetem helyi termelési és jövedelmi hatásai. Felsőoktatási Mühely 6 (3) : 33-40.

DUSEK T, LUKOVICS M (2011) Analysis of the economic impact of the Budapest Airport on the local economy. 58th Annual North American Meetings of the Regional Science Association International (RSAI), Miami, Florida, USA

FLORAX R (1992) The university: a regional booster? Avebury.

GAGNOL L, HÉRAUD J-A (2001) Impact économique régional d'un pôle universitaire : application au cas strasbourgeois. Revue d'Économie Régionale et Urbaine 2001-4 : 581-604.

GARRIDO-YSERTE R, GALLO-RIVERA M-T (2010) The impact of the university upon local economy: three methods to estimate demand-side effects. Annals of Regional Science 44: 39-67.

Hermannsson K, MCGregor P, SWALES J-K (2013) Consumption Expenditures in Economic Impact Studies: An Application to University Students. Sire Discussion Paper, SIRE-DP-2013-62. 
HuGGINS R, COOKE P (1997) The economic impact of Cardiff University: innovation, learning and job generation. Geojournal 41(4): 325-337.

JOHNSON T-M (1994) Estimating the Economic Impact of a College or University on a Nonlocal Economy. PhD dissertation, Texas Tech University.

JONGBLOED B (2008) Indicators for mapping university-regional interactions. Paper for the ENID-PRIME Indicators Conference in Oslo, 26-28 May 2008.

KLOPHAUS R (2008) The impact of additional passengers on airport employment: The case of German airports. Airport Management 2(3): 265-274.

KotOsz B (2013) The Local Economic Impact of Higher Education Institutions in Hungary. In: KHAVAND K-J (ed) Intellectual Capital Management. Global Perspectives on Higher Education, Science and Technology. IICM, Zanjan: 45-60.

Kotosz B, Gaunard-Anderson M-F, Lukovics M (2016) A Szegedi Tudományegyetem és az Université de Lorraine helyi gazdaságra gyakorolt hatásának számszerüsítése. In : LENGYEL I, NAGY B (eds) Térségek versenyképessége, intelligens szakosodása és újraiparosodása. JATEPress, Szeged : 240-254.

Kotosz B, LukOvics M, MOlNár G, ZUTI B (2015) How to measure local economic impact of universities? Methodological overview. Regional Statistics 5(2): 3-19.

LAMBOOY J (1996) Knowledge production, organisation and agglomeration economies. Tinbergen Institute discussion paper. Amsterdam, The Netherlands.

LENGYEL I (2004) Egyetemek szerepe a helyi gazdaságfejlesztésben. In: LENGYEL I (ed) A Szegedi Tudományegyetem lehetöségei a tudásalapú helyi gazdaságfejlesztésben. Kutatási háttéranyagok IV. kötet. Egyetemek szerepe a helyi gazdaság-és vállalkozásfejlesztésben. Szeged : 1-54.

LESLIE L, LEWIS D (2001) Economic Magnet and Multiplier Effects of the University of Minnesota. University of Arizona and University of Minnesota.

LU A-V, Perron R (2013) L'inscription territoriale de I'Université de Reims Champagne-Ardenne. INSEE flash Champagne-Ardenne 174.

LUKOVICS M, DUSEK T (2014) Economic Impact Analysis of the ELI R\&D Infrastructure and Science Park. Journal Mittelforum and Next Europe 1(1): 72-85

LUKOVICS M, ZUTI B (2013) Successful universities towards the improvement of regional competitiveness: „Fourth Generation" universities. Paper presented at the "European Regional Science Association (ERSA) 53th Congress „Regional Integration: Europe, the Mediterranean and the World economy" 53th Congress of the European Regional Science Association, Palermo, Italy.

LUKOVICS M, ZUTI B (2014) Egyetemek a régiók versenyképességének javításáért: „negyedik generációs” egyetemek? Tér és Társadalom 4: 77-96.

MARTIN F (1998) The economic impact of Canadian university R\&D. Research Policy 27: 677-687.

MEZEI K (2005) A Pécsi Tudományegyetem hatása a város gazdaságára. A magyar városok kulturális gazdasága. MTA Társadalomkutató Központ, Budapest.

MILLE M (2004) Université, externalités de connaissance et développement local : I'expérience d'une université nouvelle. Politiques et gestion de l'enseignement supérieur 16 (3) : 89-113.

OCDE (2007) Enseignement supérieur et régions : Concurrence mondiale engagement locale. OCDE, Paris.

PAWLOWSKI K (2009) The 'fourth generation university' as a creator of the local and regional development. Higher Education in Europe 34 (1) : 51-64.

PellenbarG P-H (2005) How to calcute the impact of the university on the regional economy. A case study of the University of Groningen. Paper presented at the Conference on Knowledge and Regional Economic Development, organised by the Regional Quantitative Analysis Research Group, University of Barcelona, 9-11 June 2005. 31 p.

POLÈSE M, LÉGER J (1979) L'impact des universités sur le développement économique régional. Conseil des universités, Québec.

REHÁK S, DŽUPKA P, ŠEBOVÁ M, SEKELSKÝ L (2015) Lokálne Ekonomické Vplyvy Univerzít. Vydavateľstvo EKONÓM, Bratislava. 
Ritsila J, Nieminen M, SOtARAuta M, LAHTONEN J (2008) L'engagement économique et social des universités en Finlande : élaboration d'un modèle d'évaluation. Politiques et gestion de l'enseignement supérieur 20 (2) : 193-214.

RYAN J, MALGIERI P (1992) Economic Impact Studies in Community Colleges: The Shortcut Method. National Council for Resource Development. Resource Paper 48, 23 p.

SEGARRA I BlASCO A (2004) La universitat com a instrument de dinamització socioconómica del territori. Coneixement i Societat 3: 78-101.

Siegfried J-J, SANDERSON A-R, MCHEnRy P (2007) The economic impact of colleges and universities. Economics of Education Review 26: 546-558.

STOKES K, COOMES P (1998) The local economic impact of higher education: an overview of methods and practice. Association for Institutional Research (AIR), Professional File 67: 16.

SZÉKELY A (2013) Regionális multiplikáció a szegedi Árkád példáján. In: RECHNITZER J, SOMLYÓDINÉ P E, KovÁcs G (eds): A hely szelleme - a területi fejlesztések lokális dimenziói. Széchenyi István Egyetem, Györ : 565-573.

VARGA A (2001) Universities and Regional Economic Development: Does Agglomeration Matter? In: Johannson B, Karlsson C, StOuGH, R (eds) Theories of Endogenous Regional Growth. Springer, Berlin.

VIDOR A (2005) A megtakarítás-ösztönzők hatása: magyarországi tapasztalatok. PM Kutatási Füzetek, Budapest.

WEST G-R, JACKSON R-W (1998) Input-output + econometric and econometric + input-output: model differences or different models? Journal of Regional Analysis and Policy 28(1): 33-48.

WISSEMA J-G (2009) Towards the third generation university. Managing the university in transition. Edward Elgar, Cheltenham.

ZUTI B, LUKOVICS M (2014) Fourth Generation Universities and Regional Development. In: HAMM R, KOPPER J (eds) Higher Education Institutions and Regional Development. Mönchengladbach : 14-31.

\section{Notes}

1 - Du point de vue de notre recherche, la nomination des différents établissements n'a aucune importance ; nous utilisons « établissement d'éducation supérieur (EES) » ou " université » pour n'importe quelle forme : école supérieure, école nationale, institut universitaire, université, etc.

2 - Les données sont accessibles à partir des systèmes d'informations internes, c'est pourquoi l'étude des écoles de commerce portugaises (Alves et al., 2015) est signée par dix-sept auteurs.

3 - On part en réalité d'une somme assez proche : 47400 francs, soit 7110 euros, ce qui n'est pas loin de 10000 euros avec l'augmentation de l'indice des prix depuis la fin des années 1990. Les auteurs ont ensuite appliqué $90 \%$ seulement dans le département du Bas-Rhin (6 400) et ont retenu finalement environ 4000 euros pour appliquer un principe de type contre factuel: une partie de ces étudiants seraient présents localement même s'il n'y avait pas d'université.

4 - Evaluation of Research Infrastructures in Open innovation and research systems, http://evario.u-strasbg.fr. 